\title{
A comparative overview of the sperm centriolar complex in mammals and birds: Variations on a theme
}

John T. Soley

Department of Anatomy and Physiology, Faculty of Veterinary Science, University of Pretoria, Private Bag X04, Onderstepoort 0110, South Africa

\section{Introduction}

Since their identification in the late nineteenth century through the pioneering work of Theodor Boveri, Edouard van Beneden, Walther Flemming and others (Bornens and Gönczy 2014; Scheer, 2014; Schatten and Sun, 2015), the structure and function of centrosomes and centrioles have intrigued scientists. An extensive amount of data has been generated on this complex organelle during the past 120 years, both in terms of its morphology and in respect of its role in cell division, ciliogenesis and fertilization. This topic has been comprehensively reviewed with more recent studies focussing on the molecular basis of centriole-centrosomal structure and dysfunction (Schatten and Sun, 2009, 2012). The ultrastructural features of the centrosome have been documented in numerous publications. It consists essentially of a pair of centrioles aligned perpendicular to each other (forming a diplosome) and surrounded by an osmiophilic, fibro-granular matrix, the pericentriolar material (PCM) (Avidor-Reiss et al., 2015; Bornens and Gönczy, 2014; Chemes, 2012; Manandhar et al., 2005; Sathananthan, 2012; Sathananthan et al., 1996). Each centriole forms a hollow cylinder, the walls of which are composed of nine circularly arranged sets of triplet microtubules (Avidor-Reiss et al., 
2015; Sathananthan, 2012; Sathananthan et al., 1996; Winey and O’Toole, 2014). In the centrosome a distinction can be made between the older (mother) centriole and the newly formed daughter centriole, although both organelles remain attached to each other (Bornens and Gönczy, 2014; Avidor-Reiss, 2015)

Early ultrastructural studies on sperm from diverse species revealed the presence of a pair of centrioles located in the neck region of the cell. This observation initiated an intense and ongoing study on the structure and role of the centrosome during spermiogenesis and fertilization (Fawcett, 1971, 1975). Much of the early work focussed on the morphology of rodent sperm (Fawcett, 1971, 1975; Fawcett and Phillips, 1969; Woolley and Fawcett, 1973), although the structural abnormalities affecting fertility in various commercially important production animals (see Chenoweth and McPherson, 2014) as well as the fine structure of human and primate sperm was also investigated (Afzelius 1996; Dadoune, 1988; Holstein and Roosen-Runge, 1981; Zamboni, 1992; Zamboni and Stefanini, 1971). In response to the growing incidence of human infertility, and prompted by the need to fully understand sperm pathologies for the application of assisted reproductive technologies (ART), research on human sperm structure expanded exponentially. In respect of the role of the centrosome in fertility studies, two focus areas soon emerged. For successful in vitro fertilisation (IVF) a thorough knowledge of sperm abnormalities was required and, based on patient studies, a number of obvious centriolar defects were identified that adversely affected fertility (Afzelius, 1996; Chemes, 2012; Chemes and Sedo, 2012; Moretti and Collodel, 2012; Zamboni, 1992). With the advent of intracytoplasmic sperm insemination (ICSI) it became clear that more subtle changes to the composition of the centrosome at a molecular level, not reflected as obvious structural anomalies, could adversely affect successful zygote formation 
and subsequent embryogenesis (Avidor-Reiss et al., 2015; Chemes, 2012; Chemes and Rawe, 2003, 2010; Sathananthan, 2012; Sathananthan et al., 1996).

Compared to the amount of data generated on mammalian sperm, very little information is currently available on this topic in birds. However, recent work on the characterisation of sperm defects in ratites (Du Plessis and Soley, 2011a,b, 2012a,b; Du Plessis et al., 2014) has highlighted the importance of understanding the role of the sperm centriolar complex (CC) in birds, particularly those of commercial value.

This paper presents an overview of the structure, function and anomalies of the sperm $\mathrm{CC}$ on a comparative basis between mammals and birds, excluding its role in fertilization and zygote formation. The information is based on selected references from the literature supplemented by original observations on sperm structure and spermiogenesis in disparate mammalian (cheetah and cane rat) and avian (ostrich, rhea and emu) species. The cheetah (Acinonyx jubatus) is listed as vulnerable in the Red List of Threatened Species of the International Union for Conservation for Nature (IUCN 2015), while the greater cane rat or grasscutter (Thryonomys swinderianus) is viewed as a potentially valuable unit of microlivestock. Ratites, in particular the ostrich (Struthio camelus) and emu (Dromaius novaehollandiae), are commercially exploited and form important niche industries world-wide. The overview is not exhaustive and the reader is referred to key references in the text for specific points of interest, particularly regarding the advances made in determining the molecular basis for centrosome dysfunction. 


\section{General features of the sperm centriolar complex}

In mammalian and avian sperm the CC or paternal centrosome (Sathananthan, 2012) consists of a pair of centrioles (a diplosome) that form the core of the complex neck region of the cell. This region constitutes the vital connection between the head and the flagellum. In addition, the diplosome initiates the formation, via the free end of the distal centriole (DC), of the axial filament complex (axoneme) which forms the motile apparatus of the tail. The concentration of pericentriolar material (PCM) that surrounds the diplosome in somatic cells is present in the form of ordered structures that contribute towards attaching the flagellum to the sperm head. The structure and development of the neck region of mammalian sperm has been described in detail (Barth and Oko, 1989; Brito et al., 2010; Fawcett, 1971, 1975; Fawcett and Phillips, 1969; Holstein and Roosen-Runge 1981; Manandhar and Sutovsky, 2007; Pesch and Bergmann, 2006; Phillips, 1974; Sathananthan, 2012; Zamboni, 1992) and recently summarised for birds (Aire, 2007, 2014; Jamieson, 2007).

During spermiogenesis in mammals and birds spermatids typically display both proximal and distal centrioles. However, in mature sperm of some species this arrangement varies. In the oscine clade of passerine birds, for example, the proximal centriole (PC) is generally considered to be absent (Jamieson, 2007) whereas no centrioles are reportedly present in rat sperm (Woolley and Fawcett, 1973). These variations clearly do not influence the motility of sperm which reinforces the observation that a basal body (effectively the DC which forms the axoneme), is not required for flagellar movement (Fawcett and Phillips, 1969).

The orientation of the centrioles may also differ. In most species the paired centrioles are typically aligned at right angles to each other, as also observed in the cane rat and cheetah 
(Fig. 1) and in ratites (Fig. 2). However, in some non-passerine birds such as the quail (Jamieson, 2007) and Guineafowl (Aire, 2014; Aire and Soley, 2003) the PC and DC lie almost inline. Why this preferential arrangement is reflected in mature sperm when the normal centriolar alignment persists in early round and elongating spermatids (Aire and Soley, 2003), remains unexplained. In some oscine birds such as the Masked weaver (Aire and Ozegbe, 2012) and Eurasian bullfinch (Birkhead et al., 2007) the PC is inclined at a $45^{\circ}$ angle to the DC. Whereas these deviations in the orientation of the PC are "normal" features in some avian species, incorrect alignment of the $\mathrm{CC}$ during spermiogenesis results in a number of sperm pathologies and is discussed below.

A distinct difference is obvious between mammals and birds in respect of the length of the centrioles. In mammals both centrioles are of similar length (approximately $0.5 \mu \mathrm{m}$ ) whereas in birds the DC is generally longer (see Jamieson 2007). This is particularly obvious in ratite and tinamou sperm where the DC reaches a length of $3.0 \mu \mathrm{m}$ and forms the core of the entire midpiece (Asa et al., 1986; Baccetti et al., 1991; Soley 1993; Du Plessis and Soley, 2011a; Du Plessis et al., 2014). (compare Figs. 1b \& 4b,c with Figs. 2c \& 3). The functional importance of this species-specific phenomenon remains unexplained but there are indications in ratites that the $\mathrm{DC}$, besides forming the axoneme, plays a role in reinforcing the midpiece region of the sperm in a similar fashion to that performed by the outer dense fibres in mammalian sperm (see below). 

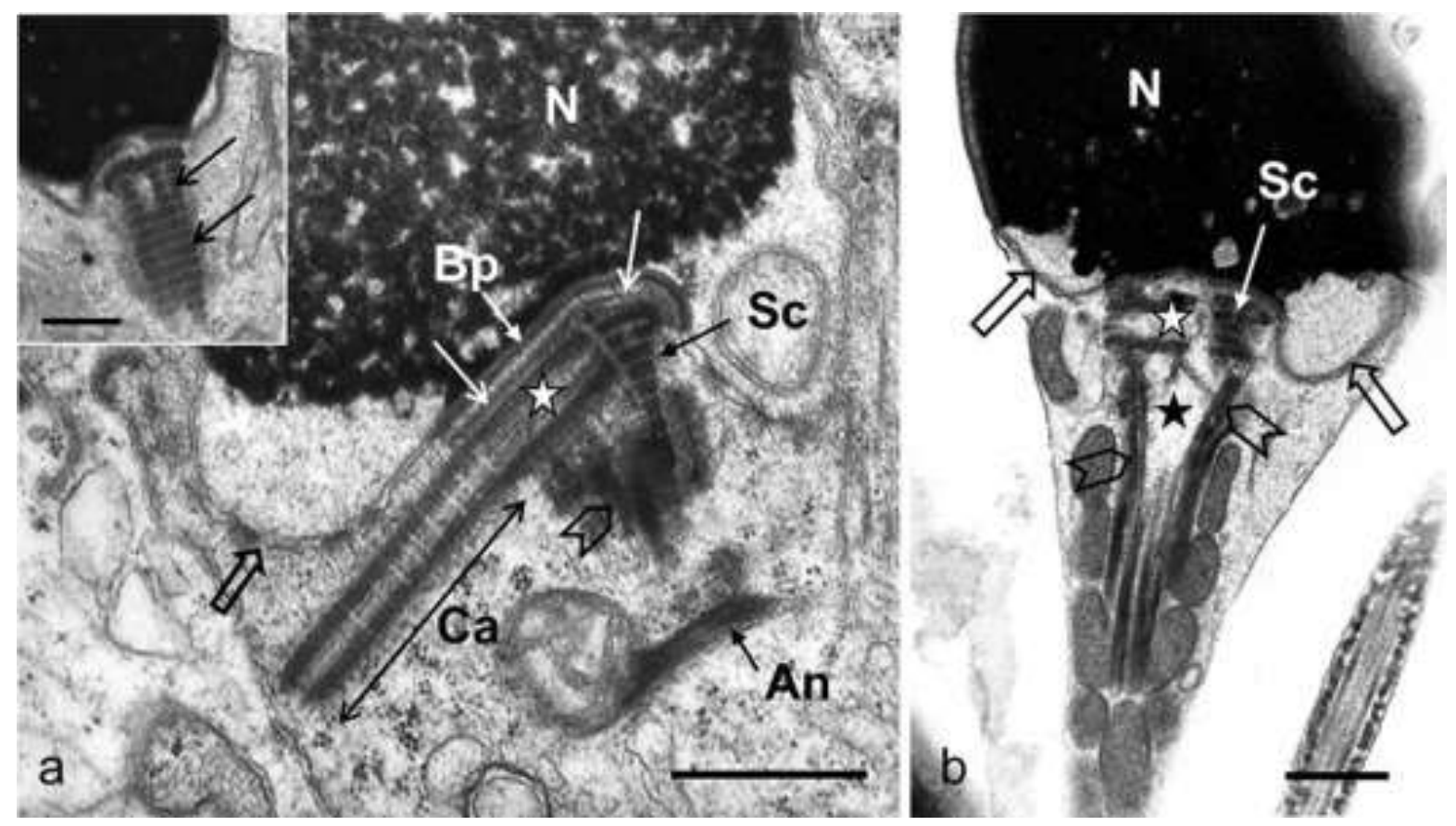

Fig. 1. (a) Neck region of a developing cheetah spermatid showing elements of the centriolar complex.

Condensing nucleus $(\mathrm{N})$, basal plate $(\mathrm{Bp})$, capitulum (arrows) proximal centriole (white star), centriolar adjunct (Ca), segmented column of the connecting piece (Sc), annulus (An), dense fibres (chevron), redundant nuclear membrane (block arrow). The distal centriole and flagellum are not in the plane of section. Inset: The centriolar complex in a mature sperm. The striated columns of the connecting piece (arrows) mask both the proximal and distal centrioles. (b) Neck region of an ejaculated cane rat sperm showing the proximal (white star) and distal (black star) centrioles, dense fibres (chevrons), redundant nuclear membrane (block arrows) and striated columns (Sc). Note the empty/disrupted appearance of the distal centriole. Bars $=0.5 \mu \mathrm{m}$.

\section{Ultrastructure of the centriolar complex}

As noted above, the $\mathrm{CC}$ is composed of a PC, DC and attendant PCM. When present, the PC in both vertebrate groups is short $[0.3 \mu \mathrm{m}$ in the ostrich (Soley, 1993) and approximately $0.5 \mu \mathrm{m}$ in mammals as in somatic cells (Agircan et al., 2014)], lies partially within the implantation fossa at the base of the nucleus, and displays the characteristic pinwheel 

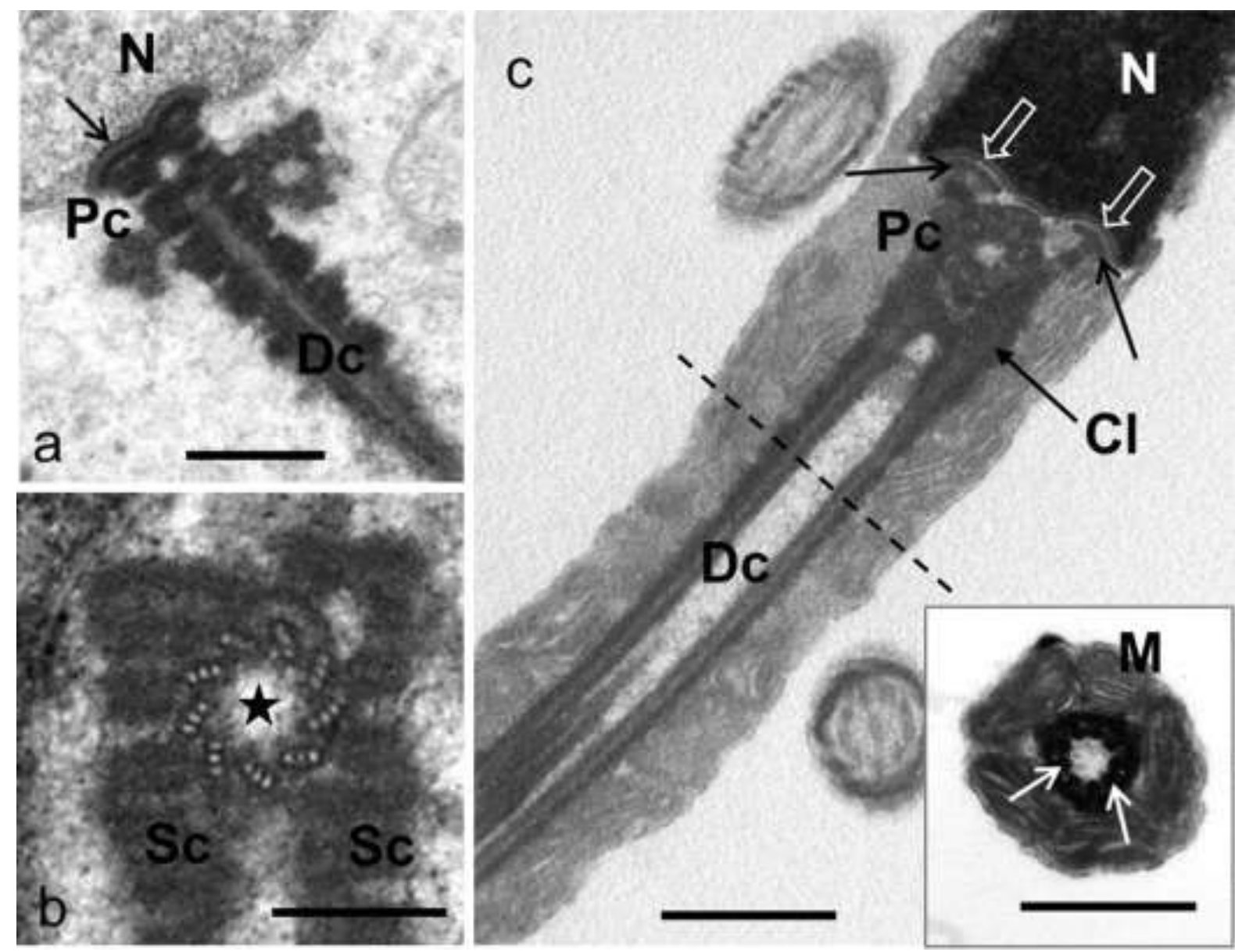

Fig. 2. (a) Early rhea spermatid showing attachment of the centriolar complex to the nucleus (N) via a shallow implantation fossa (arrow). The segmented nature of the pericentriolar material surrounding the proximal (Pc) and anterior aspect of the distal (Dc) centrioles is obvious. (b) A later stage ostrich spermatid displaying the typical pinwheel arrangement of the nine sets of microtubular triplets in the wall of the proximal centriole. Note the empty appearance of the centriolar lumen (star) and the segmented nature of the forming columns of the connecting piece $(\mathrm{Sc})$. (c) Neck and anterior midpiece of a mature emu sperm. The centriolar complex consists of the proximal (Pc) and distal (Dc) centrioles, the columns ( $\mathrm{Cl}$ ) and capitulum (arrows) of the connecting piece and the basal plate (block arrows). The anterior segment of the distal centriole displays an empty lumen.

Nucleus (N). Inset: A transverse section through the distal centriole at the level indicated by the dashed line in Fig. 2c. Dense fibres (arrows) jut from the wall of the centriole into the lumen. Mitochondria (M). Bars (a), (c), inset $=0.5 \mu \mathrm{m}$, bar $(\mathrm{b})=0.25 \mu \mathrm{m}$.

arrangement of microtubular triplets embedded in a ring of moderately electron-dense material (Avidor-Reiss et al., 2015; Du Plessis and Soley, 2014; Sathananthan, 2012; Soley, 
1993) (Fig. 2b). It is surrounded by small amounts of centrosomal proteins including $\gamma$ tubulin, centriole-associated centrin and other components, the precise functions of which remain undetermined (Comizzoli and Wildt, 2012; Schatten and Sun, 2011, 2012; Schatten et al., 2011). The lumen of the PC shows no elaborations, generally appearing "empty" or containing fine, flocculent material. In mammalian sperm the proximal (and distal) centriole is completely surrounded peripherally by a series of nine segmented (striated) columns that merge above the centriole to form a conspicuous layer of material, the capitulum (Fawcett, 1975) (Fig. 1).These structures are collectively referred to as the connecting piece (Fig. 1). In birds the columns enclosing the PC are non-segmented (Fig. 2c) although during spermiogenesis in ratites the segmented nature of the peri-centriolar material is a distinctive feature of the developing spermatids (Fig.2a,b). The narrow distal aspect of the segmented columns merges with the nine outer dense fibres of the midpiece in mammalian sperm although this association is not obvious in avian sperm. A typical capitulum is reportedly absent in birds (Aire, 2014) although in ratites this component of the connecting piece is well-developed and separated from the basal plate lining the implantation fossa by an intervening layer of clear material. As in mammals (Fawcett and Phillps, 1969), fine, perpendicularly oriented filaments traverse this layer effecting attachment of the sperm head to the flagellum. In mammalian spermatids a distinctive lateral extension of the PC is observed which has been termed the centriolar adjunct (Fig. 1a) (Fawcett and Phillips, 1969). This structure has been described as a tuft of microtubules possibly indicative of abortive formation of an axoneme by the PC (De Krester and Kerr, 1994) and may be observed in mature sperm (Sathananthan et al., 1996). A centriolar adjunct has not been identified in birds although this term has been used to describe a granular structure which appears in early spermatids in passerine birds. This structure is not associated with the CC and is more aptly described as the "granular body" or "granular helix" (Aire, 2014). 
During the early stages of spermiogenesis in mammals the twin centrioles of the diplosome display similar structural features. However, in later stage spermatids species specific disintegration of the centrioles occurs, a process referred to as centrosome reduction (Manandhar et al., 2005). This process involves not only disintegration of the centrioles but also the selective loss of centrosomal proteins. In rodents (rat and mouse) both centrioles are lost but in various other mammals the PC remains unaffected while the DC displays varying degrees of disintegration (see Manandhar et al., 2005). It is interesting to note that the PC appears to be lost in oscines in contrast to the situation in most mammals where the DC is affected. Prior to centrosome reduction, the DC, together with the PC, assists in the formation of the segmented columns of the connecting piece (Fawcett and Phillips, 1969) and also gives rise to the axonemal microtubules (see below) (Sathananthan, 2012). However, during later stages of spermiogenesis the DC undergoes varying degrees of disintegration. These changes include ballooning of the centriole, disruption and partial loss of the triplet microtubules, and extension of the central pair of axonemal microtubules throughout the length of the centriolar lumen (Fawcett and Phillips, 1969; Manandhar et al., 2005; Sathananthan, 2012). These features are typically seen in cheetah sperm but in the cane rat the central pair of microtubules are seldom visible in the centriolar lumen (Figs. 1b, 4c, 5a,b). In contrast, the DC of avian sperm, which varies in length, retains its structure (Jamieson, 2007) in mature sperm. In ratites and the tinamou the wall of the DC is a more substantial structure and consists of a relatively narrow ring of moderately electron-dense material from which nine evenly spaced dense projections jut into the centriolar lumen, and between which are located the nine sets of triplet microtubules (inset Fig. 2c) (Soley, 1993). These projections reportedly represent the nine outer dense fibres present in the midpiece and principal piece of mammalian sperm (Jamieson, 2007). In most non-passerine birds the lumen of the DC 
remains empty (Jamieson, 2007) but in ratites the central pair of axonemal microtubules, as in mammalian sperm, occupy much of its length. This is noteworthy considering the length $(3 \mu \mathrm{m})$ of the DC in these species. In the emu, for example, the central microtubules are generally not present in the proximal third of the DC (Fig. 2c). Unlike the situation in mammals the central microtubules are embedded in a rod of dense material (see Fig. 3b).

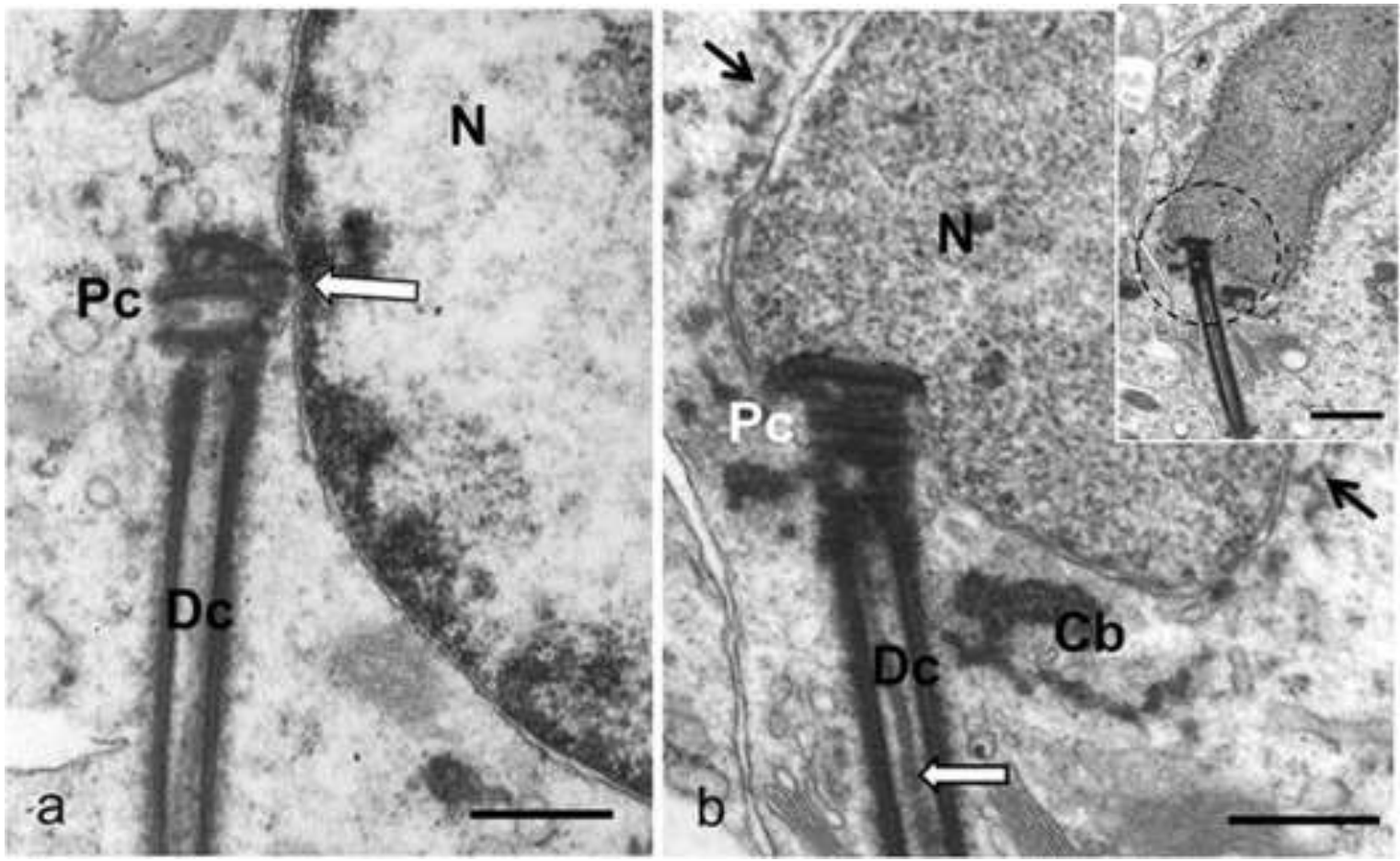

Fig. 3. (a) Early ostrich spermatid showing misalignment of the centriolar complex with respect to the nucleus $(\mathrm{N})$. Although the proximal centriole $(\mathrm{Pc})$ lies close to the nuclear membrane, the nucleolemma remains unmodified and does not form an implantation fossa (arrow). Distal centriole (Dc). (b) An elongating ostrich spermatid demonstrating abaxial attachment of the centriolar complex to the base of the nucleus $(\mathrm{N})$. Proximal centriole $(\mathrm{Pc})$, distal centriole $(\mathrm{Dc})$ containing a rod of dense material (block arrow), chromatoid body $(\mathrm{Cb})$, microtubules of the circular manchette (arrows). Inset: Low power electron micrograph to illustrate the degree of misplacement of the $\mathrm{CC}$ from the sperm head axis. Bars $=0.5 \mu \mathrm{m}$. 


\section{Function}

As an entity the $\mathrm{CC}$ has the obvious functions of forming the flagellum and effecting its attachment to the sperm head during spermiogenesis. Additionally, the CC plays an essential role in mitotic spindle formation in the developing zygote (Chemes, 2012). Data emanating from research on various sperm pathologies have pointed to a significant degree of autonomy between the proximal and distal centrioles (Chemes, 2012), stressing the importance of each component of the diplosome in sperm function.

Both centrioles appear to be involved in the recruitment of pericentriolar material which is converted into the segmented columns and capitulum of the connecting piece (Fawcett, 1971). This is certainly true for mammalian sperm but in many avian species, particularly in ratites, involves only the most anterior part of the distal centriole owing to its great length. However, in both vertebrate groups analogous structures are formed that effectively form a cohesive connection between the head and tail. The fusion of the segmented columns of the connecting piece with the outer dense fibres of the flagellum would firmly connect the two components. The segmented or striated nature of the mammalian connecting piece would also lend a degree of flexibility to the neck region, particularly as the contact between head and tail constitutes a firm connection as opposed to an articulation. The similarity in structure between the connecting piece and ciliary rootlets which anchor the basal bodies in the ectoplasm of ciliated cells (Fawcett, 1971) further emphasises the important role of the CC in firmly binding the head to the tail. In birds the connecting piece, which appears less complex in design (Aire, 2007, 2014; Jamieson, 2007), would presumably fulfil a similar function despite its amorphous form. It has been reported that the actual mode of contact between the head and tail resides in the fine filaments that traverse the space between the capitulum and 
basal plate lining the implantation fossa (Fawcett and Phillips, 1969; Fawcett, 1975). In birds the attachment of the centrioles to the nucleus appears to be effected by amorphous material that lines the implantation fossa (Aire, 2014).

The function of the PC is to migrate and attach correctly to the spermatid nucleus. Defective migration or abnormal implantation of the diplosome via the PC results in a number of sperm pathologies (Chemes and Rawe, 2010) common to mammals and birds and are described below. The PC also acts as a microtubule organising centre (MTOC) for the zygote following fertilization by recruiting pericentriolar material (PM) which will contribute towards the mitotic spindle (Chemes, 2012). To fulfil this function the PC must be freed from the surrounding elements of the connecting piece, a process which may be facilitated by the proteasomes present in the sperm neck region (for relevant references see Chemes, 2012). The decrease in proteasome activity in acephalic or abnormally aligned sperm (Chemes and Rawe, 2010) and resultant impairment of normal centriole function in the zygote would support the role of proteasomes in releasing the PC. These observations further emphasises the need to understand the molecular mechanisms underlying centriolar structure and function in both sperm and zygote (Avidor-Reiss et al., 2015).

The DC, before its reduction in mammalian sperm, serves to initiate the formation of axonemal microtubules during the early stages of spermiogenesis. This is achieved by "the assembly of axoneme doublet microtubules by direct tubulin nucleation on subunits $\mathrm{a}$ and $\mathrm{b}$ of distal centriolar triplets" (Chemes, 2012). Failure to generate normal axonemes results in a number of sperm defects implicated in severe asthenozoospermia (see below). The outer dense fibres (ODF) in mammalian sperm that run through the midpiece and part of the principal piece have been credited with reinforcing the structure of the flagellum and acting 
as force multipliers to enhance the torque generated by the axonemal microtubules.

Numerous proteins associated with their assembly have been identified (Hermo et al., 2010), suggesting that these fibres are involved in more complex functions than merely reinforcing the flagellar axoneme (Chemes and Rawe, 2010). Whereas dense fibres are present to varying degrees in both passerine and non-passerine birds (Jamieson, 2007) and would presumably perform a similar function to those in mammals, only rudimentary fibres are found in the proximal principal piece of palaeognathous birds. However, the specific structure of the DC in these species, which runs the length of the midpiece, would suggest that the ODF's are incorporated into the wall of the centriole as also seen in crocodiles (Jamieson, 2007). In ratites, therefore, the fibres would certainly strengthen the structure of the midpiece but would not function as force multipliers as they do not surround the axoneme in these species. The close structural association between the ODF's and the segmented columns, capitulum and basal plate of the connecting piece is demonstrated at the molecular level by their common complement of the $84 \mathrm{kDa}$ protein (Schalles et al., 1998).

\section{Structural anomalies associated with the centriolar complex}

The link between sperm neck defects and asthenozoospermia has been demonstrated in a number of publications and the negative impact of these defects on successful intracytoplasmic sperm injection (ICSI) highlighted (Chemes, 2000, 2012; Chemes et al., 1999; Rawe et al., 2002). Moreover, an association between abnormal alignment of the headflagellum junction and poor centrosomal function has been established (Comizzoli and Wildt, 2012; Manandhar et al., 2005) which adversely affects fertilization or impairs development of the zygote (Moretti and Collodel, 2012). Additionally, failure of the DC to initiate a normal 
axoneme also results in deficient sperm motility (Andersson et al., 2000; Hrudka et al., 1991). Whereas the range of anomalies associated with the $\mathrm{CC}$ has been grouped as a specific syndrome for human sperm (Chemes, 2000, 2012; Chemes et al., 1999; Chemes and Sedo, 2012; Dadoune, 1988; Holstein et al., 1988; Moretti and Collodel, 2012; Sathananthan, 2012; Zamboni, 1992) less comprehensive or encompassing descriptions are available for other mammals (Andersson et al., 2000; Barth and Oko, 1989; Blom and Birch-Andersen, 1970; Brito, 2007; Hrudka et al., 1991; Oettlé and Soley, 1988). In birds, this syndrome has only been described in any detail in ratites (Du Plessis and Soley, 2011a,b,2012a,b; Du Plessis et al., 2014) while studies on poultry are silent on the topic.

In the most extreme situation the $\mathrm{CC}$ fails to migrate towards the nucleus, despite appearing morphologically normal and generating a flagellum via the DC. The sperm head is subsequently retained and phagocytosed by the Sertoli cells (Chemes et al., 1999) while the tail is freed during spermiation. This defect has been observed in man (Chemes et al., 1999, Moretti and Collodel, 2012) and other mammals (Blom and Birch-Andersen, 1970; Toyama and Itoh, 1996) and referred to as acephalic (decapitated) sperm. The headless tail displays swelling of the neck region and varying degrees of development and arrangement of the CC and mitochondrial sheath. Similar features have been observed in the cheetah and cane rat and are also obvious, although the incidence is low, in ratites (Fig. 4f). This anomaly is reflected in early spermatids by an obvious gap between the nucleus and $\mathrm{CC}$, resulting in eventual separation of the two components. An interesting observation in the ostrich and emu, and also reported in man (Chemes et al., 1999) is that in some instances the CC approaches the nuclear membrane (normal migration) but fails to attach, seemingly due to a lack of formation of the implantation fossa and concurrent modification of the nucleolemma (Fig.3a). In yet other cases the detachment between head and tail can occur between the two 

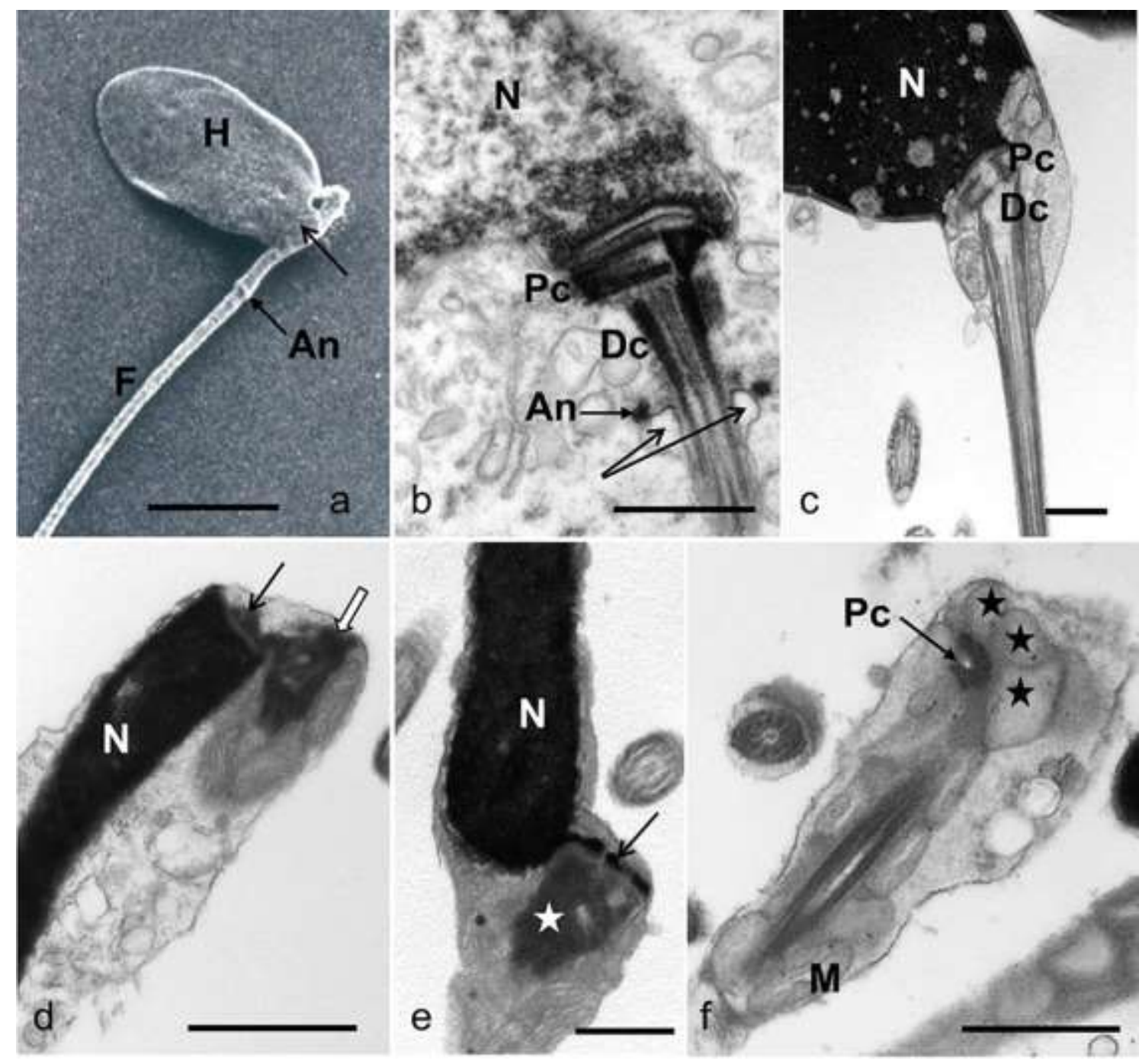

Fig. 4. (a) Scanning electron micrograph of a cane rat sperm. Note the $90^{\circ}$ alignment between the head $(\mathrm{H})$ and flagellum (F) and lack of correct attachment of the centriolar complex (arrow) which lies at right angles to the head base. Annulus (An). (b) A transmission electron micrograph of a cane rat spermatid demonstrating the same anomalous head/tail alignment shown in Fig.4a. In this instance the centriolar complex has attached to the nucleus $(\mathrm{N})$ but is incorrectly oriented. Proximal centriole (Pc), distal centriole (Dc), annulus (An), flagellar canal (arrows). (c) Mature cane rat sperm demonstrating both abaxial attachment of the centriolar complex to the head base and incorrect alignment of approximately $45^{\circ}$. Proximal centriole (Pc), distal centriole (Dc), nucleus (N). (d) Disjointed ostrich sperm. The flagellum is reflected at an angle of $180^{\circ}$ leaving elements of the connecting piece lodged within the implantation fossa (arrow) and around the centriolar complex (block arrow). Nucleus (N). (e) An example of abaxial tail attachment in an emu sperm. The ill-defined centriolar complex (star) attaches to an extension of the nucleus (arrow) a distance from the sperm head axis. Nucleus (N). (f) An oblique section of an acephalic sperm in the cane rat. The proximal centriole $(\mathrm{Pc})$ is the only obvious component 
of the centriolar complex which is separated from its point of attachment to the nucleus by nuclear membrane derived vacuoles (stars). Mitochondria of the midpiece $(M)$. Bar $(a)=5 \mu \mathrm{m}$; bars $(b-f)=0.5 \mu \mathrm{m}$.

centrioles or at the posterior aspect of the nucleus (Afzelius, 1996). The aberrant positioning of cell organelles (eg. mitochondria) or vacuoles between the nucleus and $\mathrm{CC}$ during spermiogenesis (see Fig. 4f) can also physically prevent contact and subsequent connection between the two components. The essential role of the protein centrobin in the assembly of the "head-tail coupling apparatus" during spermiogenesis points to a possible molecular basis for the formation of acephalic sperm (Liška et al., 2009). Work on the factors responsible for positioning of the centrosome in somatic cells may also shed further light on this phenomenon (Agircan et al., 2014).

A variant of this condition involves the centrosome migrating to the caudal pole of the nucleus but without adopting the normal linear orientation. This results in lateral positioning of the head at various angles (as great as $180^{\circ}$ ) to the neck/midpiece (Afzelius, 1996; Brito et al., 2010; Chemes and Sedo, 2012; Moretti and Collodel, 2012; Rawe et al., 2002). Both cane rat (Figs. $4 \mathrm{a}-\mathrm{c}$ ) and cheetah sperm exhibit this range of angled tail attachments and a similar situation has been described in the ostrich (Du Plessis et al., 2014) and emu (Du Plessis and Soley, 2011a). A particular form of this defect, characterised by complete separation of the neck from the head-base at the connecting piece with a $180^{\circ}$ reflex of the tail, which aligns the head and tail parallel to each other but within the confines of the plasmalemma, has been described in both ratite species (Du Plessis and Soley, 2011b; Du Plessis et al., 2014). The specific point of separation of the two components appeared to occur in the vicinity of the implantation fossa (Fig. 4d), leaving the CC coupled to the detached tail. The term “disjointed sperm" has been used to describe this defect in ratites. This particular anomaly 
has been described, for example, in man (Chemes and Rawe, 2003; Zamboni, 1992) and mouse (Mochida et al., 1999), and is speculated to result from structural deficiencies and/or deficiencies in the assembly of periaxonemal proteins during spermiogenesis, causing the dislocation of the segmented columns of the connecting piece from the nuclear attachment sites (Mochida et al., 1999). To what extent the DC of ratites, which often appears forced to adopt an angled approach to the nucleus due to its length, plays a role in this phenomenon, remains to be determined.

Another manifestation of the failure of the $\mathrm{CC}$ to attach normally to the caudal pole of the nucleus is that of abaxial sperm. This defect involves the eccentric positioning of the $\mathrm{CC}$ relative to the longitudinal axis of the sperm head and has been described in several mammalian species (see Du Plessis and Soley, 2012a), including man (Chemes and Rawe, 2010). In spatulate mammalian sperm the broad head base generally accommodates abaxial attachment of the flagellum without elements of the $\mathrm{CC}$ overlapping the nuclear base (Fig.4c). However, in ratites the similarity in width of the CC and head base (see Jamieson, 2007), coupled with the abaxial positioning of the CC, results in a staggered alignment of the head and tail in defective sperm (Du Plessis and Soley, 2012a). A phenomenon observed in ratites is the formation of a tenuous nuclear extension to accommodate the misaligned CC (Fig. 4e). In birds and mammals the misalignment of the CC manifests during the early elongation phase of spermiogenesis (Fig. 3b) but is probably initiated during attachment of the $\mathrm{CC}$ to the nucleus in round spermatids. Despite the obvious morphological differences between avian and mammalian sperm, a similar mode of formation of abaxial sperm is therefore apparent. It should be noted, however, that in most stallion sperm the implantation fossa and basal plate are eccentric in position with respect to the breadth of the cell, and thus sperm with abaxial tail are considered normal in this species (Brito, 2007). Barth and Oko 
(1989) also report an association between abaxial sperm and rudimentary tails in the bull, a condition also observed in the American black bear (Brito et al., 2010.)

Failure of the DC to form a morphologically normal axoneme results in poor sperm motility or immotility. The most obvious manifestation of this form of centriolar defect is reflected in the "stump tail" or "short tail" sperm defect, the distinction being made in terms of the length of the flagellum in affected cells (Andersson et al., 2000). This defect has been described in various mammalian species (Andersson et al., 2000; Hrudka et al., 1991) and irrespective of the length of the stunted tail, a common feature is the disruption of the normal $9 \times 2+2$ arrangement of the axonemal microtubules, in particular the loss of one or both of the central singlet microtubules (Andersson et al., 2000; Hrudka etal., 1991). According to Hrudka et al. (1991) this defect "seems associated with the inability of the DC to implant on the plasmalemma, to produce the axoneme, or maintain its growth, and to induce the normal differentiation of periaxonemal structures". Other syndromes in man that point towards defective functioning of the DC include primary ciliary diskinesia (PCD) and dysplasia of the fibrous sheath (DFS). In both instances, in addition to features typical for each syndrome, missing axonemal central pairs and lack of dynein arms on the microtubular doublets, are characteristic features indicative of a loss of centriolar function (Chemes, 2000; Chemes and Rawe, 2010; Chemes and Sedo, 2012; Chemes et al., 1998; Rawe et al., 2001).

Whereas the above-mentioned defects arise from defective migration or orientation of the $\mathrm{CC}$, abnormal duplication of the centrioles during spermatogenesis results in the formation of multiflagellate sperm. This relatively rare condition may adversely influences sperm motility and therefore fertility (Barth and Oko, 1989; Escalier, 1983; Nistal et al., 1977). The ultrastructural features of this defect have been thoroughly described, demonstrating a degree 


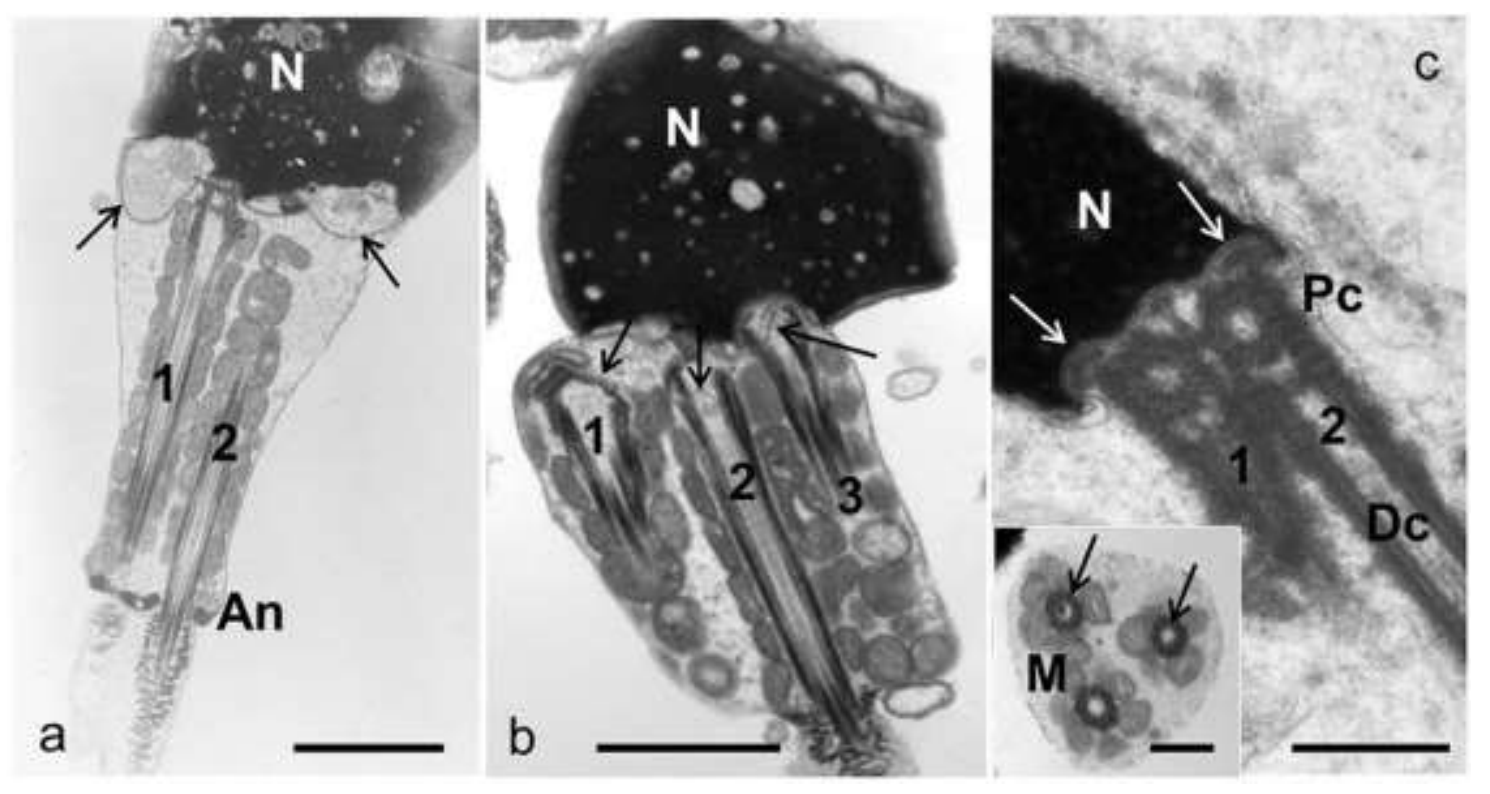

Fig. 5. (a) Planar section of a cane rat sperm with twin flagella (1 and 2). Flagellum 1 shows the proximal and empty distal centriole which is not in the plane of section in flagellum 2. Nucleus $(\mathrm{N})$, redundant nuclear membrane (arrows), annulus (An). (b) Planar section of a cane rat sperm displaying both multiple flagella (1, 2 and 3) and abaxial placement of flagellum 1 and 2. The proximal centrioles and surrounding elements of the connecting piece are absent or poorly developed (arrows). Flagellum 1 makes no contact with the nucleus (N). (c) Longitudinal section of a rhea sperm with a double tail. Note the duplicated centriolar complexes (1 and 2) and the extensive implantation fossa (arrows). Proximal centriole (Pc), distal centriole (Dc). Inset: Transverse section of a triple flagellum in an emu sperm. The centriolar complex is sectioned through the distal centriole (arrows) which forms the core of the midpiece in each of the tails. Mitochondria (M). Bars $=0.5 \mu \mathrm{m}$.

of uniformity acrossvarious mammalian species (Barth and Oko 1989; Brito, 2007; Brito et al., 2010; Escalier 1983; Nistal et al., 1977), and non-passerine birds (see Du Plessis and Soley, 2012b). The neck region of affected sperm reveal two or more apparently morphologically normal centriolar complexes (Fig. 5a,c), although disruption of the connecting piece and absence of the PC may occur (Fig. 5b). The supernumerary CC's are generally each housed within a separate implantation fossa which would seem to suggest that the $\mathrm{PC}$ and surrounding peri-centriolar material is responsible for initiating the localised transformation of the nucleolemma into the fossa. Each CC generates a typical flagellum of 
which the principal pieces remain free in contrast to the collectively bound midpieces (Fig. 5). Unlike the situation in mammals where the broad nuclear base generally provides enough space for the implantation of multiple CC's, in bird sperm of normal dimensions the narrow, cylindrical head base displays tenuous peripheral widening in order to accommodate the additional diplosomes (Du Plessis and Soley, 2012b). In multiple tailed human sperm, the axonemal complexes often appear to be defective (Escalier, 1983; Nistal et al., 1977) as also sometimes observed in the cane rat (see Fig. 5b). However, in ratites the CC's appeared morphologically normal (Fig. 5c).

Two possible explanations have been proposed for the origin of multiple CC's. The relatively high proportion of macrocephalic sperm with multiple tails in both mammals and birds is suggestive of disrupted meiotic division during spermatogenesis and the resultant production of diploid and polyploid cells (Barth and Oko, 1989; Kopp et al., 2007; Perrin et al., 2008). Abnormal cycles of centriole formation would similarly result in multiflagellate sperm (Escalier 1983).

\section{Conclusion}

Although the basic design of the CC is similar between birds and mammals, certain differences are apparent. Centriole reduction does not generally occur in birds, but when present as in oscines, involves the loss of the PC. The structure of the avian connecting piece is less complex and the mode of attachment between head and tail at the implantation fossa appears to differ. Based on morphological similarities and the mode of development during spermiogenesis, the individual components of the $\mathrm{CC}$ would appear to share comparable 
functions. Faulty development, migration and alignment of the $\mathrm{CC}$ during spermiogenesis is reflected in a number of characteristic sperm anomalies which share common expression despite the obvious differences in sperm head shape between mammals (spatulate) and birds (vermiform), hence, variations on a theme. A comparison of defective sperm formation and centrosomal dysfunction at the molecular level is currently difficult owing to the paucity of relevant information on avian sperm.

\section{Acknowledgments}

The technical expertise and intellectual inputs provided by Dr Lizette du Plessis, without whose assistance this comparative study would not have been possible, are gratefully acknowledged. The University of Pretoria is thanked for financial assistance.

\section{References}

Afzelius, B.A., 1996. The sperm cytoskeleton and its defects. Cytoskeleton 3, 325-357.

Aire, T.A., 2007. Spermatogenesis and testicular cycles, in: Jamieson, B.M.G. (Ed.), Reproductive Biology and Phylogeny of Birds Vol 6A. Science Publishers, Jersey, pp. 279347.

Aire, T.A., 2014. Spermiogenesis in birds. Spermatogenesis 4:3 e959392. 
Aire, T.A., Ozegbe, P.C., 2012. Components and development of the centriolar complex during and beyond spermiogenesis in a passerine bird, the Masked Weaver (Ploceus velatus). Tiss. Cell 44, 63-67.

Aire, T.A., Soley, J.T., 2003. The Guinea fowl spermatozoon centriolar complex: A morphological deviation for a non-passerine bird. Proc. Microsc. Soc. South. Afr. 33, 75.

Agircan, F.G., Schiebel, E., Mardin, B.R., 2014. Separate to operate: control of centrosome positioning and separation. Phil. Trans. R. Soc. B 369, 20130461.

Andersson, M., Peltoniemi, O.A.T., Mäkinen, A., Sukura, A., Rodriguez-Mártinez, H., 2000. The hereditary 'short tail' sperm defect - a new reproductive problem in Yorkshire boars. Reprod. Dom. Anim. 35, 59-63.

Asa, C., Phillips, D.M., Stover, J., 1986. Ultrastructure of spermatozoa of the crested tinamou. J. Ultra. Mol. Struct. R. 94,170-175.

Avidor-Reiss, T., Khire, A., Fishman, E.L., Jo, K.H., 2015. Atypical centrioles during sexual reproduction. Front. Cell Dev. Biol. 3, 21.

Baccetti, B., Burrini, A.G., Falchetti, E., 1991. Spermatozoa and relationships in paleognath birds. Biol. Cell 71, 209-216.

Barth, A.D., Oko, R.J., 1989. Abnormal morphology of bovine spermatozoa. Iowa State University Press. 
Birkhead, T.R., Giusti, F., Immler, S., Jamieson, B.G.M., 2007. Ultrastructure of the unusual spermatozoon of the Eurasian bullfinch (Pyrrhula pyrrhula). Acta Zool. (Stockholm) 88, 119-128.

Blom, E., Birch-Andersen, A., 1970. Ultrastructure of the decapitated sperm defect in Guernsey bulls. J. Reprod. Fert. 23, 67-72.

Bornens, M., Gönczy, P., 2014. Centrosomes back in the limelight. Phil. Trans. R. Soc. B $369,20130452$.

Brito, L.M.C. 2007. Evaluation of Stallion Sperm Morphology. Clin. Tech. Equine Pract. 6,249-264.

Brito, L.F.C., Sertich, P.L., Stull, G.B., Rives, W., Knobbe, M. 2010. Sperm ultrastructure, morphometry, and abnormal morphology in American black bears (Ursus americanus) Theriogenology 74, 1403-1413.

Chemes, H.E., 2000. Phenotypes of sperm pathology: Genetic and acquired forms in infertile men. J. Androl. 21, 799-808.

Chemes, H. E., 2012. Sperm centrioles and their dual role in flagellogenesis and cell cycle of the zygote, in: Schatten, H. (Ed.), The Centrosome. Humana Press, New York, pp. 33-48. 
Chemes, H.E., Brugo Olmedo, S., Carrere, C., Oses, R., Carizza, C., Leisner, M., Blaquier, J., 1998. Ultrastructural pathology of the sperm flagellum: association between flagellar pathology and fertility prognosis in severely asthenozoospermic men. Hum. Reprod. 13, $2521-2526$.

Chemes, H.E., Puigdomenech, E.T., Carizza, C., Brugo Olmedo, S., Zanchetti, F., Hermes, A., 1999. Acephalic spermatozoa and abnormal development of the head-neck attachment. A human syndrome of genetic origin. Hum. Reprod. 14, 1811-1818.

Chemes, H.E., Rawe, V.Y. 2003. Sperm pathology: a step beyond descriptive morphology. Origin, characterization and fertility potential of abnormal sperm phenotypes in infertile men. Hum. Reprod. Update 9, 405-428.

Chemes,H.E., Rawe,V.Y., 2010.The making of abnormal spermatozoa: cellular and molecular mechanisms underlying pathological spermiogenesis. Cell Tissue Res. 341, 349357.

Chemes, H.E., Sedo, C.A., 2012. Tales of the tail and sperm head aches. Asian J. Androl. 14: $14-23$.

Chenoweth, P.J., McPherson, F.J., 2014. Genetic aspects of male reproduction, in: Chenoweth, P.J., Lorton, S.P. (Eds.), Animal Andrology: Theories and Applications. CAB International, Boston, pp. 144-173. 
Comizzoli, P., Wildt, D.E. 2012. Centrosomal Functions and Dysfunctions in Cat Spermatozoa, in: Schatten, H. (Ed.), The Centrosome. Humana Press, New York, pp. 49-58.

Dadoune, J.P., 1988. Ultrastructural abnormalities of human spermatozoa. Hum. Reprod. 3, 311-318.

De Kretser, D.M., Kerr, J.B., 1994. The cytology of the testis, in: Knobil, E., Neill, J.D. (Eds.), The physiology of reproduction, $2^{\text {nd }}$ edition. Raven Press, New York, pp. 1177-1290.

Du Plessis, L., Malecki, I., Bonato, M., Smith, M., Cloete, S., Soley, J. 2014. A morphological classification of sperm defects in the ostrich (Struthio camelus). Anim. Reprod. Sci. 150, 130-138.

Du Plessis, L., Soley, J.T., 2011a. Incidence, structure and morphological classification of abnormal spermatozoa in the emu (Dromaius novaehollandiae). Theriogenology 75, 589-601.

Du Plessis, L., Soley, J.T., 2011b. Head-base bending and disjointed spermatozoa in the emu (Dromaius novaehollandiae): a morphological comparison of two closely related defects. Theriogenology 76, 1275-1283.

Du Plessis, L., Soley, J.T., 2012a. Abaxial tail implantation in the emu, Dromaius novaehollandiae: morphological characteristics and origin of a rare avian sperm defect. Theriogenology 77, 1137-1143. 
Du Plessis, L., Soley, J.T., 2012b. Structural peculiarities associated with multiflagellate sperm in the emu, Dromaius novaehollandiae. Theriogenology 78, 1094-1101.

Du Plessis, L., Soley, J.T. 2014. A re-evaluation of sperm ultrastructure in the emu, Dromaius novaehollandiae. Theriogenology 81, 1073-1084.

Escalier, D., 1983. Human spermatozoa with large heads and multiple flagella: a quantitative ultrastructural study of 6 cases. Biol. Cell 48, 65-74.

Fawcett, D.W., 1971. Observations on cell differentiation and organelle continuity in spermatogenesis, in: Beatty, R.A., Gluecksohn-Waelsch, S. (Eds.), Proc. Int. Symp. The genetics of the spermatozoon. Edinburgh. pp. 37-68.

Fawcett, D.W., 1975. The mammalian spermatozoon. Dev. Biol. 44, 394-436.

Fawcett, D.W., Phillips, D.M., 1969. The fine structure and development of the neck region of the mammalian spermatozoon. Anat. Rec. 165, 153-184.

Hermo, L., Pelletier, R-M., Cyr, D, Smith, C.E., 2010. Surfing the wave, cycle, life history, and genes/proteins expressed by testicular germ cells. Part 3: Developmental changes in spermatid flagellum and cytoplasmic droplet and interaction of sperm with the zona pellucida and egg plasma membrane. Microsc. Res. Techniq. 73, 320-363.

Holstein, A.F., Roosen-Runge, E.C., 1981. Atlas of Human Spermatogenesis. Grosse Verlag, Berlin. 
Holstein, A.F., Roosen-Runge, E.C., Schirren, C., 1988. Illustrated pathology of human spermatogenesis. Grosse Verlag, Berlin.

Hrudka, F., Betsch, J.M., Kenney, R.M., 1991. Anomalies of centriolar derivatives manifest in spematic flagella and respiratory cilia of the stallion. Arch. Androl. 27, 161-175.

IUCN 2015. The IUCN Red List of Threatened Species. Version 2015-3.

http://www.iucnredlist.org. Downloaded on 9 September 2015.

Jamieson, B.G.M., 2007. Avian spermatozoa: structure and phylogeny, in: Jamieson, B.G.M. (Ed.), Reproductive Biology and Phylogeny of Birds Part A. Science Publishers, Enfield (NH), USA, pp. 349-511.

Kopp, C., Sukura, A., Tuunainen, E., Gustavsson, I., Parvinen, M., Andersson, M., 2007. Multinuclear-multiflagellar sperm defect in a bull - a new sterilizing sperm defect. Reprod. Domest. Anim. 42, 208-213.

Liška, F., Gosele, C., Rivkin, E., Tres,L., Cardoso, M.C., Domaing,P., Krejčı, E., Šnajdr,P., Lee-Kirsch, M.A., de Rooij, D.G., Křen, V., Křenovà , D., Kierszenbaum, A.L., Hubner, N., 2009. Rat hd mutation reveals an essential role of centrobin in spermatid head shaping and assembly of the head-tail coupling apparatus. Biol. Reprod. 81, 1196-1205.

Manandhar G., Schatten, H., Sutovsky, P., 2005 Centrosome reduction during gametogenesis and its significance. Biol. Reprod. 72, 2-13. 
Manandhar, G., Sutovsky, P., 2007. Comparative Histology and Subcellular Structure of Mammalian Spermatogenesis and Spermatozoa, in: Schatten, H., Constantinescu, G.M. (Eds.), Comparative Reproductive Biology, Blackwell Publishing Ltd, Oxford, pp. 81-98.

Mochida, K., Tres, L.L., Kierszenbaum, A.L., 1999. Structural and Biochemical Features of Fractionated Spermatid Manchettes and Sperm Axonemes of the Azh/Azh Mutant Mouse. Mol. Reprod. Dev. 52, 434-444.

Moretti, E., Collodel, G., 2012. Electron microscopy in the study of human sperm pathologies, in: Méndez-Vilas, A. (Ed). Current Microscopy Contributions to Advances in Science and Technology, pp. 343-351.

Nistal, M., Paniagua, R., Herruzo, A., 1977. Multi-tailed spermatozoa in a case with asthenospermia and teratospermia. Virchows Arch. B 26,111-118.

Oettlé, E.E., Soley, J.T., 1988. Sperm abnormalities in the dog: A light and electron microscopic study. Vet. Med. Rev. 59, 28-70.

Perrin, A., Morel, F., Moy, L., Colleu, D., Amice, V., De Braekeleer, M., 2008. Study of aneuploidy in large headed, multiple-tailed spermatozoa: case report and review of the literature. Fertil. Steril. 90,13-17.

Pesch, S., Bergmann, M., 2006. Structure of mammalian spermatozoa in respect to viability, fertility and cryopreservation. Micron 37, 597-612. 
Phillips, D.M., 1974. Spermiogenesis. Academic Press, New York.

Rawe, V.Y., Galaverna, G.D., Acosta, A.A., Brugo Olmedo, S., Chemes, H.E., 2001.

Incidence of tail structure distortions associated with dysplasia of the fibrous sheath in human spermatozoa. Hum. Reprod. 16, 879-886.

Rawe, V., Terada, Y., Nakamura, S., Chillik, C.F., Brugo Olmedo, S., Chemes, H.E., 2002. A pathology of the sperm centriole responsible for defective sperm aster formation, sygamy and cleavage. Hum. Reprod. 17, 2344-2349.

Sathananthan, A.H., 2012. Human centrosomal dynamics during gametogenesis, fertilization, and embryogenesis and its impact on fertility: ultrastructural analysis, in: H. Schatten (Ed.), The Centrosome. Humana Press, New York, pp. 73-97.

Sathananthan, A.H., Ratnam, S.S., Ng, S.C., Thrin, J.J., Gianaroli, L., A.Trounson, A., 1996. The sperm centriole: its inheritance, replication and perpetuation in early human embryos. Hum. Reprod. 11, 345-356.

Schalles, U., Shao, X., van der Hoorn, F.A., Oko, R., 1998. Developmental expression of the 84-kDa ODF sperm protein: Localization to both the cortex and medulla of outer dense fibers and to the connecting piece. Dev. Biol. 199, 250-260.

Schatten, H., Rawe, V.Y., Sun, Q-Y., 2011. The sperm centrosome: its role and significance in nature and human assisted reproduction. J. Reprod. Stem Cell Biotechnol. 2, 121-127. 
Schatten, H., Sun, Q-Y., 2009. The functional significance of centrosomes in mammalian meiosis, fertilization, development, nuclear transfer, and stem cell differentiation. Environ. Mol. Mutagen. 50, 620-636.

Schatten, H., Sun, Q-Y., 2011. New insights into the role of centrosomes in mammalian fertilization and implications for ART. Reproduction 142, 793-801.

Schatten, H., Sun, Q-Y., 2012. Nuclear-centrosome relationships during fertilization, cell division, embryo development, and in somatic cell nuclear transfer embryos, in: Schatten, H. (Ed.), The Centrosome. Humana Press, New York, pp. 59-72.

Schatten, H., Sun, Q-Y., 2015. Centrosome and microtubule functions and disfunctions in meiosis: implications for age-related infertility and developmental disorders. Reprod. Fert. Develop. 27, 934-943.

Scheer, U., 2014. Historical roots of centrosome research: discovery of Boveri's microscope slides in Würzburg. Phil. Trans. R. Soc. B 369, 20130469.

Soley, J.T., 1993. Ultrastructure of ostrich (Struthio camelus) spermatozoa: I. Transmission electron microscopy. Onderstepoort J. Vet. Res. 60, 119-130.

Toyama, Y., Itoh, Y., 1996. Ultrastructural features and pathogenesis of decapitated spermatozoa in a boar. Andrologia 28, 109-115. 
Winey, M., O’Toole, E., 2014. Centriole structure. Phil. Trans. R. Soc. B 369, 20130457.

Woolley, D.M., Fawcett, D.W., 1973. The degeneration and disappearance of the centrioles during the development of the rat spermatozoon. Anat. Rec. 177, 289-302.

Zamboni, L., 1992. Sperm structure and its relevance to infertility. Arch. Pathol. Lab. Med. $116,325-344$.

Zamboni, L., Stefanini, M., 1971. The fine structure of the neck of mammalian spermatozoa. Anat. Rec. 169, 155-172. 